\title{
Study on the Unified Mathematical Model for All Types of Cam Mechanisms
}

\author{
Wei $\mathrm{He}^{1,2, \mathrm{a}}$, Xueming $\mathrm{He}^{2, \mathrm{~b} *}$, Feng $\mathrm{Xu}^{1, \mathrm{c}}$, Jiujun Zhen ${ }^{1, \mathrm{~d}}$, Rong Zhang ${ }^{3, \mathrm{e}}$ \\ ${ }^{1}$ Mechanical Engineering Department of Nanjing Institute of Industry Technology, Nanjing 210023, \\ China \\ ${ }^{2}$ Jiangsu Key Laboratory of Advanced Food Manufacturing Equipment and Technology, Jiangnan \\ University, Wuxi, 214122, China \\ ${ }^{3}$ School of Science, Jiangnan University, Wuxi, 214122, China \\ ahewei1957@163.com, ${ }^{b}$ hxueming@163.com, ${ }^{c} x u f @ n i i t . e d u . c n, ~{ }^{d}$ zhengij@niit.edu.cn, \\ ezhr89@163.com
}

Keywords: mechanism, cam, curve, curved surface, CAD

\begin{abstract}
This paper presents the unified mathematical model well applicable for the analysis and design of the planar and spatial cam mechanisms of all categories in terms of the differential geometry and meshing principle, exploring the interrelation of the various cam mechanisms. The general-purpose CAD/CAM software package for cam mechanisms is made possible by utilizing the unified mathematical approach developed in this paper. The normalization of the initial parameters for the CAD of certain representative cam mechanisms is also examined.
\end{abstract}

\section{Notations}

C constant vector

$C_{1} \quad$ transformation vector of translation

from $S_{\mathrm{f}}$ to $\mathrm{S}_{1}$

$C_{2} \quad$ transformation vector of translation

from $\mathrm{S}_{2}$ to $\mathrm{S}_{\mathrm{c}}$

$E^{j \theta_{2}}, E^{-k \theta_{1}}$ rotation matrices for the rotation transformations of $\mathrm{S} 2$ about the $\mathrm{j}$ axis and $\mathrm{S}_{\mathrm{f}}$ about the $\mathrm{k}$ axis

$h_{1} \quad$ follower displacement

$h_{2}$ cam displacement

$h_{1 \mathrm{x}}, h_{1 \mathrm{y}}, h_{1 \mathrm{z}}$ components of any follower displacement $\mathrm{h}_{1}$ in space in the direction $\mathrm{x}_{\mathrm{f}}, \mathrm{y}_{\mathrm{f}}$ and $\mathrm{z}_{\mathrm{f}}$

$J_{1}, J_{2}$ 2nd and the 3rd component of the rotation matrix

$j \quad$ axis $\mathrm{x}_{2}$ or $\mathrm{y}_{2}$ or $\mathrm{z}_{2}$

$k \quad$ axis $\mathrm{x}_{1}$ or $\mathrm{y}_{1}$ or $\mathrm{z}_{1}$

$P \quad$ contact point

$\boldsymbol{R}_{\mathrm{f}} \quad$ vector notation of $\Sigma_{1}$ in $\mathrm{S}_{\mathrm{f}}$

$\boldsymbol{R}_{1} \quad$ vector notation of $\Sigma_{1}$ in $\mathrm{S}_{1}$

$\boldsymbol{R}_{2} \quad$ vector notation of $\Sigma_{1}$ in $S_{2}$

$\boldsymbol{R}_{\mathrm{C}} \quad$ vector notation of $\Sigma_{1}$ in $\mathrm{S}_{\mathrm{c}}$

$r_{\mathrm{f}}$ follower rotation radius

$r_{0}=r_{0}\left(\delta_{\mathrm{f}}\right) \quad$ element function of a specific rotating curved surface

$\mathrm{S}_{1}\left(\mathrm{o}_{1}, \mathrm{x}_{1}, \mathrm{y}_{1}, \mathrm{z}_{1}\right) \quad$ fixed system on the initial position of follower.

$\mathrm{S}_{\mathrm{f}}\left(\mathrm{o}_{\mathrm{f}}, \mathrm{x}_{\mathrm{f}}, \mathrm{y}_{\mathrm{f}}, \mathrm{z}_{\mathrm{f}}\right) \quad$ motive system on the follower with the initial position lying on $\mathrm{S}_{1}$.

$\mathrm{S}_{2}\left(\mathrm{O}_{2}, \mathrm{X}_{2}, \mathrm{y}_{2}, \mathrm{Z}_{2}\right)$ fixed system on the initial position of cam, whose direction is consistent with $\mathrm{S}_{1}$.

$\mathrm{S}_{\mathrm{c}}\left(\mathrm{o}_{\mathrm{c}}, \mathrm{x}_{\mathrm{c}}, \mathrm{y}_{\mathrm{c}}, \mathrm{z}_{\mathrm{c}}\right) \quad$ motive system on cam, whose initial position lies on $\mathrm{S}_{2}$. 
$t \quad$ time

$\boldsymbol{V}_{1}, \boldsymbol{V}_{2}$ translatory velocities of follower and cam.

$\Sigma_{1} \quad$ mating surface of follower

$\Sigma_{2} \quad$ cam curved surface (curve)

$\varphi, \delta_{\mathrm{f}} \quad$ curved vein coordinates of the roller curved surface.

$\beta \quad$ constant angle, $\beta=0^{\circ}$ or $\beta=90^{\circ}$

$\delta_{\mathrm{fx}}, \delta_{\mathrm{fy}}, \delta_{\mathrm{fz}} \quad$ curve vein coordinates of $\sum_{1}$

$\theta_{1}, \theta_{2} \quad$ angular displacements of follower and cam

$\phi \quad$ contact function, $\phi\left(\delta_{f}, \varphi, t\right)=0$

$\omega_{1}, \omega_{2} \quad$ angular velocities of follower and cam

$\rho_{1}, \rho_{2},-\rho_{3} \quad$ components of $\mathrm{O}_{1} \mathrm{O}_{2}$ in the direction of $\mathrm{x}_{1}, \mathrm{y}_{1}$ and $\mathrm{z}_{1}$, respecti

\section{Introduction}

The traditional design and manufacture of cam mechanisms have not met the requirements of industry. Although the CAD/CAM technology of cams develops rapidly, nevertheless a number of CAD software for cam mechanisms find their limited application to some extent due to a great diversity and variation of cam mechanisms. This paper makes an approach to the development of the unified mathematical model, the design and the analysis of cam mechanisms.

\section{Formulation of unified mathematical model}

(a)Universal geometric model of cam mechanisms

The implement of motion specifications of cam mechanisms is dependent on the cam profile or the shape of working curved surface and the arrangement of mechanism configuration. For this reason, it is necessary to formulate the mathematical model of the cam profile or the curved surface while we proceed to the precise design of cam mechanisms by using the CAD technique. First of all, a universal geometric model for cam mechanism is constructed. As shown in Figure 1, the geometric model is represented by the kinematics schematic diagram of the spatial cam mechanism that is most general. This model can be converted to the planar or spatial cam mechanisms with the diverse configuration and the forms of motion after the different initial parameters are chosen. The four different Cartesian coordinate systems are established in Figure 1 to derive the accurate mathematical formula of cam profile or the working curved surface of cam:

$\mathrm{S}_{1}\left(\mathrm{o}_{1}, \mathrm{x} 1, \mathrm{y} 1, \mathrm{z} 1\right)$ - $\mathrm{a}$ fixed system on the initial position of follower.

$\mathrm{S}_{\mathrm{f}}\left(\mathrm{o}_{\mathrm{f}}, \mathrm{x}_{\mathrm{f}}, \mathrm{y}_{\mathrm{f}}, \mathrm{z}_{\mathrm{f}}\right)$ - a motive system on the follower with the initial position lying on $\mathrm{S}_{1}$.

$\mathrm{S}_{2}\left(\mathrm{O}_{2}, \mathrm{X}_{2}, \mathrm{y}_{2}, \mathrm{z}_{2}\right)$ - a fixed system on the initial position of cam , whose direction is consistent with $S_{1}$.

$\mathrm{S}_{\mathrm{c}}\left(\mathrm{o}_{\mathrm{c}}, \mathrm{X}_{\mathrm{c}}, \mathrm{yc}, \mathrm{zc}\right)$ - a motive system on cam, whose initial position lies on $\mathrm{S}_{2}$

On the basis of the envelope theory of differential geometry, the coordinate transformation can be made by utilizing the rotation matrix or the translation of coordinates in such a manner that the vector notation of the mating surface $\Sigma_{1}$ of follower in $S_{f}$ relative to the contact point $P$ is transformed to that in $\mathrm{S}_{\mathrm{c}}$ relative to the cam working curved face as the roller curved faces of the family as the envelope surface. Then the contact equation can be derived by dint of meshing principle (contact condition) again, resulting in the unified mathematic expression for the cam-curved surface (curve) $\sum_{2}$, that is, $\Sigma_{1} \stackrel{l(p)}{\longrightarrow} \sum_{2}$. The coordinate transformation of vector is

$\boldsymbol{R}_{\mathrm{f}}^{(P)} \rightarrow \boldsymbol{R}_{1}{ }^{(P)} \rightarrow \boldsymbol{R}_{2}{ }^{(P)} \rightarrow \boldsymbol{R}_{\mathrm{c}}{ }^{(P)}$

where these vectors above can be simply rewritten as: $\boldsymbol{R}_{\mathrm{f}}, \boldsymbol{R}_{1}, \boldsymbol{R}_{2}$, and $\boldsymbol{R} \boldsymbol{c}$. 


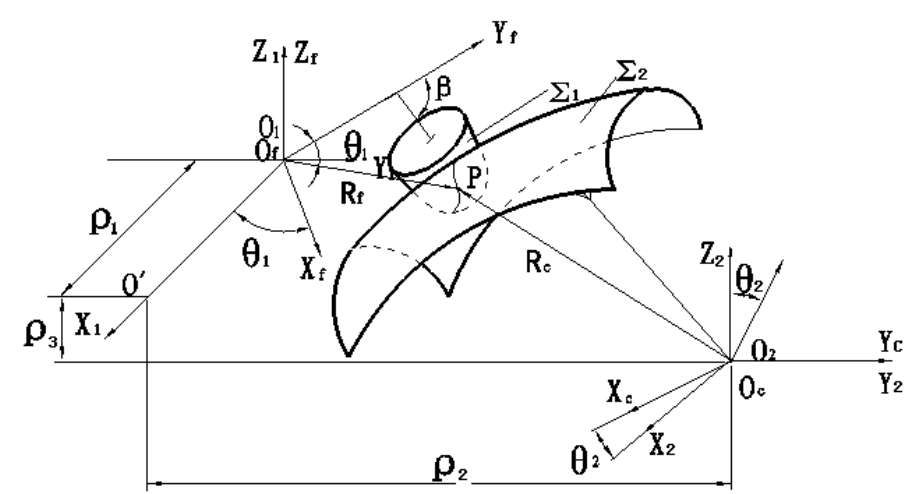

Fig.1 The universal geometric model of cam mechanism

(b)Vector notation $\boldsymbol{R}_{\mathrm{f}}$ of $\Sigma_{1}$ in $\mathrm{S}_{\mathrm{f}}$

There are many forms of engagement or contact between a cam curved surface and a follower in direct contact, such as the roller, the flat-faced, the knife-edge, etc. Among them the roller follower is most complicated, consisting of the cylindrical roller, the conic roller, the spherical roller and the other kinds of rollers with rotating curved face, etc. However, no matter what classes of roller, $\Sigma_{1}$ is usually a given simple curved face .The mathematical expression of vector $\boldsymbol{R}_{\mathrm{f}}$ in $\mathrm{S}_{1}$ is generally simple.

Let $\sum_{1}$ be any specific rotation curved surface with the roller follower. As the rotating of roller about its axis has no effect upon the forms of engagement, the roller can be supposed to be fixed on the follower as shown in Figure 2. In this case, the vector $\boldsymbol{R}_{\mathrm{f}}$ for $\Sigma_{1}$ is as follows

$$
\boldsymbol{R}_{\mathrm{f}}=\left\{\begin{array}{l}
\mathrm{x}_{\mathrm{f}} \\
\mathrm{y}_{\mathrm{f}} \\
\mathrm{z}_{\mathrm{f}}
\end{array}\right\}=\left\{\begin{array}{l}
r_{0} \sin \varphi \\
\left(\delta_{\mathrm{f}}+b\right) \cos \beta+r_{0} \cos \varphi+r_{\mathrm{f}} \\
-\left(\delta_{\mathrm{f}}+b\right) \sin \beta
\end{array}\right\}
$$

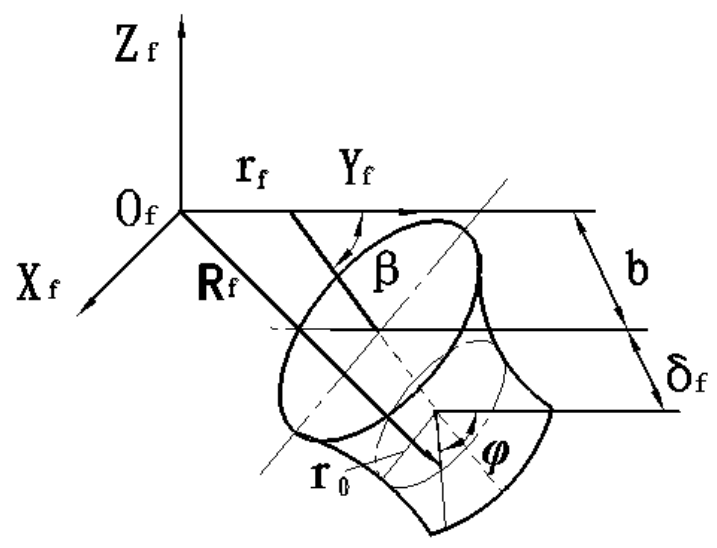

Fig. 2 Vector $\boldsymbol{R}_{\mathrm{f}}$ for roller curved surface $\Sigma_{1}$

where $r_{0}=r_{0}\left(\delta_{\mathrm{f}}\right)$ is the element function of a specific rotating curved surface , $\varphi$ and $\delta_{\mathrm{f}}$ represent the curved vein coordinates of the roller curved surface.

Since the follower is in the translational motion for the flat-faced follower, $\beta$ is either 0 or $90^{\circ}$.As a result, when $\beta=0^{\circ}$,

$$
\boldsymbol{R}_{\mathrm{f}}=\left\{\begin{array}{l}
\mathrm{x}_{\mathrm{f}} \\
\mathrm{y}_{\mathrm{f}} \\
\mathrm{z}_{\mathrm{f}}
\end{array}\right\}=\left\{\begin{array}{l}
\delta_{\mathrm{fx}} \\
r_{\mathrm{f}}+b \\
\delta_{\mathrm{fz}}
\end{array}\right\}
$$

and when $\beta=90^{\circ}$, 


$$
\boldsymbol{R}_{\mathrm{f}}=\left\{\begin{array}{l}
\mathrm{x}_{\mathrm{f}} \\
\mathrm{y}_{\mathrm{f}} \\
\mathrm{z}_{\mathrm{f}}
\end{array}\right\}=\left\{\begin{array}{l}
\delta_{\mathrm{fx}} \\
\delta_{\mathrm{fy}} \\
b
\end{array}\right\}
$$

where $\delta_{\mathrm{fx}}$ and $\delta_{\mathrm{fz}}$ or $\delta_{\mathrm{fx}}$ and $\delta_{\mathrm{fy}}$ are the curve vein coordinates of $\sum_{1}$ For the knife-edge follower, $r_{0}=\delta_{\mathrm{f}}=0$, therefore, $\boldsymbol{R}_{\mathrm{f}}$ is a constant vector:

$$
\boldsymbol{R}_{\mathrm{f}}=\left\{\begin{array}{l}
\mathrm{x}_{\mathrm{f}} \\
\mathrm{y}_{\mathrm{f}} \\
\mathrm{z}_{\mathrm{f}}
\end{array}\right\}=\left\{\begin{array}{l}
0 \\
b \cos \beta+r_{\mathrm{f}} \\
b \sin \beta
\end{array}\right\}
$$

The $\boldsymbol{R}_{\mathrm{f}}$ above are also suitable for the planar cam mechanisms. In this instance, assuming $z_{\mathrm{f}}=0$ and $\beta=0, \boldsymbol{R}_{\mathrm{f}}$ can be reduced to the two-dimensional vectors. It must be noted that although only the roller follower was previously illustrated for a clear visualization's sake, the model shown in Figure 1 is likewise suited for the other forms of contact.

(c) Transformation of coordinates

In order to derive the mathematical formula of $\boldsymbol{R}_{\mathrm{c}}$, the transformation of the four coordinate systems from $S_{f}$ to $S_{c}$ will be necessarily made. The transformation of the coordinate from $S_{0}$ to $S_{1}$ depends on the motion form of follower in addition that the transformation from $S_{1}$ to $S_{2}$ needs to be added a constant vector $\boldsymbol{C}$. On the other hand, the coordinate transformation of $S_{2}$ to $S_{c}$ depends on the motion form of cam. In spite of the fact that there are a wide variety of forms in the motion of cam mechanisms, the unified mathematical model can be obtained from the following several vectors $\boldsymbol{R}_{\mathrm{c}}$.

(1) If the cam rotates about the $j$ axis (axis $x_{2}$ or $y_{2}$ or $z_{2}$ ) and the follower rotates about the $k$ axis (axis $\mathrm{x}_{1}$ or $\mathrm{y}_{1}$ or $\mathrm{z}_{1}$ ), then

$$
\boldsymbol{R}_{\mathrm{C}}=E^{j \theta_{2}}\left(E^{-k \theta_{1}} \boldsymbol{R}_{\mathrm{f}}-\boldsymbol{C}\right)
$$

where $E^{j \theta_{2}}, E^{-k \theta_{1}}$ are the rotation matrices for the rotation transformations of $S_{2}$ about the $j$ axis and $\mathrm{S}_{\mathrm{f}}$ about the $\mathrm{k}$ axis, $\theta_{1}$ and $\theta_{2}$ are the angular displacements of follower and cam, respectively.

(2) If the cam rotates about the $\mathrm{j}$ axis and the follower translates (in-line), then

$\boldsymbol{R}_{\mathrm{C}}=E^{j \theta_{2}}\left(\boldsymbol{R}_{\mathrm{f}}+\boldsymbol{C}_{1}-\boldsymbol{C}\right)$

where $\boldsymbol{C}_{1}=\boldsymbol{C}_{1}\left(h_{1}\right)$ is a transformation vector of translation from $\mathrm{S}_{\mathrm{f}}$ to $\mathrm{S}_{1}, \boldsymbol{h}_{1}$ is the follower displacement.

(3) If the cam translates and the follower rotates about the $\mathrm{k}$ axis, then

$\boldsymbol{R}_{\mathrm{c}}=\left(E^{-k \theta_{1}} \boldsymbol{R}_{\mathrm{f}}-\boldsymbol{C}\right)-\boldsymbol{C}_{2}$

where $-C_{2}=-C_{2}\left(h_{2}\right)$ is a transformation vector of translation from $\mathrm{S}_{2}$ to $\mathrm{S}_{\mathrm{c}}, h_{2}$ is the cam displacement.

(4) If the cam translates and the follower translates (in-line), then

$\boldsymbol{R}_{\mathrm{C}}=\left[\left(\boldsymbol{R}_{\mathrm{f}}+\boldsymbol{C}_{1}\right)-\boldsymbol{C}\right]-\boldsymbol{C}_{2}$

These classes discussed above are the most common forms in the motion of cam mechanisms. Especially, when the cam contains more complex motions, i.e. both the rotary and the translating one, the additional cases results:

(5) If the cam rotates and translates, and the follower rotates or oscillates about the k axis, then

$\boldsymbol{R}_{\mathrm{C}}=E^{j \theta_{2}}\left(E^{-k \theta_{1}} \boldsymbol{R}_{\mathrm{f}}-\boldsymbol{C}\right)-\boldsymbol{C}_{2}$

(6) If the cam rotates and translates, and the follower translates, then

$\boldsymbol{R}_{\mathrm{C}}=E^{j \theta_{2}}\left[\left(\boldsymbol{R}_{\mathrm{f}}+\boldsymbol{C}_{1}\right)-\boldsymbol{C}\right]-\boldsymbol{C}_{2}$

Summarily, the most general motion forms of cam mechanisms can be boiled down to the following: cam and follower rotate or oscillate and translate. Such motion forms will cover all kinds of the motions described previously, and so the unified vector expression for the cam curved faces or the curves can be constructed as

$$
\boldsymbol{R}_{\mathrm{C}}=E^{j \theta_{2}}\left[\left(E^{-k \theta_{1}} \boldsymbol{R}_{\mathrm{f}}+\boldsymbol{C}_{1}\right)-\boldsymbol{C}\right]-\boldsymbol{C}_{2}
$$

As the kinematical parameters will be given the different values according to the corresponding 
motion forms, Equation (4) can be converted to the previous expression of $\boldsymbol{R}_{\mathrm{c}}$. For example, as in cam or follower without translating motion, $h_{2}$ or $h_{1}$ will equal 0 , so $\boldsymbol{C}_{2}$ or $\boldsymbol{C}_{1}$ becomes a zero vector. In case of cam or follower without rotary motion, $\theta_{1}$ or $\theta_{2}$ will equal 0 , so $E^{j \theta_{2}}$ or $E^{-k \theta_{1}}$ can be turned into an identity matrix. In this situation, either the zero vectors or the identity matrix has no influence on the $\boldsymbol{R}_{\mathrm{C}}$ specified by Equation (4). It follows that Equation (4) can represent the cam curved surfaces or the curves of cam mechanisms with a number of motion forms.

In relation to the planar mechanism, since $\boldsymbol{R}_{\mathrm{c}}$ lies in the $\mathrm{x}-\mathrm{y}$ plane, the rotation matrix will become

$$
\begin{gathered}
\left\{\begin{array}{c}
E^{j \theta_{2}}=\left[\begin{array}{ll}
\cos \theta_{2} & \sin \theta_{2} \\
-\sin \theta_{2} & \cos \theta_{2}
\end{array}\right] \\
E^{-k \theta_{1}}=\left[\begin{array}{ll}
\cos \theta_{1} & -\sin \theta_{1} \\
\sin \theta_{1} & \cos \theta_{1}
\end{array}\right]
\end{array}\right. \\
\text { if } \theta_{1}=\theta_{2}=0 \text {, then } \\
E^{j \theta_{2}}=E^{-k \theta_{1}}=\left[\begin{array}{ll}
1 & 0 \\
0 & 1
\end{array}\right]
\end{gathered}
$$

(d)Contact conditions

In addition, since the cam and the follower must satisfy the contact condition at the mating point $\mathrm{P}$, the unified mathematical formula expressed by Equation (4) remains incomplete. According to the meshing theory, a fixed position on the follower is in contact with the corresponding counterpart on the cam at each time $t$, which indicates that $\theta_{2}, \theta_{1}, h_{2}$ and $h_{1}$ are all the function of $t$. From the envelope theory, it is known that the cam-curved surface is a single parameter envelope surface of the curved face of follower with respect to $t$, and the contact conditions (contact equations) are:

$$
\begin{array}{ll}
\phi\left(\delta_{\mathrm{f}}, \varphi, t\right)=0 & \text { for spatial mechanisms } \\
\phi(\varphi, t)=0 & \text { for planar mechanisms }
\end{array}
$$

that is

$$
\phi\left(\delta_{\mathrm{f}}, \varphi, t\right)=\left[\frac{\partial \boldsymbol{R}_{\mathrm{c}}}{\partial \delta_{f}}, \frac{\partial \boldsymbol{R}_{\mathrm{c}}}{\partial \varphi}, \frac{\partial \boldsymbol{R}_{\mathrm{c}}}{\partial t}\right]=0
$$

The mixing product of the three vectors $\frac{\partial \boldsymbol{R}_{\mathrm{c}}}{\partial \delta_{\mathrm{f}}}, \frac{\partial \boldsymbol{R}_{\mathrm{c}}}{\partial \varphi}, \frac{\partial \boldsymbol{R}_{\mathrm{c}}}{\partial t}$ is zero.

$$
\phi(\varphi, t)=\left|\frac{\partial \boldsymbol{R}_{\mathrm{c}}}{\partial \varphi} \times \frac{\partial \boldsymbol{R}_{\mathrm{c}}}{\partial t}\right|=0
$$

where

$$
\left\{\begin{array}{l}
\frac{\partial \boldsymbol{R}_{\mathrm{c}}}{\partial \delta_{\mathrm{f}}}=E^{j \theta_{2}} E^{-k \theta_{1}} \frac{\partial \boldsymbol{R}_{\mathrm{f}}}{\partial \delta_{\mathrm{f}}} \\
\frac{\partial \boldsymbol{R}_{\mathrm{c}}}{\partial \varphi}=E^{j \theta_{2}} E^{-k \theta_{1}} \frac{\partial \boldsymbol{R}_{\mathrm{f}}}{\partial \varphi} \\
\frac{\partial \boldsymbol{R}_{\mathrm{c}}}{\partial}=\omega_{2} J_{2} E^{j \theta_{2}}\left[\left(E^{-k \theta_{1}} \mathrm{R}_{\mathrm{f}}+\boldsymbol{C}_{1}\right)-\boldsymbol{C}\right]-\boldsymbol{V}_{2}+E^{j \theta_{2}}\left(-\omega_{1} J_{3} \mathrm{R}_{\mathrm{f}}+\boldsymbol{V}_{1}\right)
\end{array}\right.
$$

In Equation (7), $\omega_{1}$ and $\omega_{2}$ are the angular velocities of follower and cam, $\boldsymbol{V}_{1}, \boldsymbol{V}_{2}$ are the translating velocities of follower and cam, respectively, $\omega_{1}, \omega_{2}, \boldsymbol{V}_{1}, \boldsymbol{V}_{2}$ embodying the motion specifications of cam mechanisms, are known, $J_{2}$ and $J_{3}$ are the 2nd and the 3rd component ${ }^{[4]}$ of the rotation matrix.

Hence, the unified mathematical model for the cam curved surfaces and the curve is completely formulated:

$$
\boldsymbol{R}_{\mathrm{C}}=E^{j \theta_{2}}\left[\left(E^{-k \theta_{1}} \boldsymbol{R}_{\mathrm{f}}+\boldsymbol{C}_{1}\right)-\boldsymbol{C}\right]-\boldsymbol{C}_{2}
$$




$$
\phi\left(\delta_{\mathrm{f}}, \varphi, t\right)=0 \text { or } \phi(\varphi, t)=0
$$

The contact conditions reflect the relationship between the curved veins coordinates of cam working curved surface at the contact point P. For each time t and any given $\delta_{\mathrm{f}}, \varphi$ can be specified. For a planar cam, when $\delta_{\mathrm{f}}$ is known as a constant and $\varphi$ is eliminated, the curve equation for cam, with $\mathrm{t}$ being a parametric variable, can be obtained.

Having formulated the unified mathematical model $(*)$ for cam profile or working curved surface, the unified formula for the pressure angle and the curvature, etc., which will no longer be discussed here, can be accordingly achieved.

\section{Normalization of the initial parameter for CAD of CAM mechanisms}

As the cam profile (or curved surface) is generated, in line with the unified mathematical model developed above, the normalization of the initial parameters must be taken into account to adapt for the diverse cam mechanisms. In other words, how to select and enter the initial parameters is a prerequisite consideration to automatically define every vector and matrix in Equation (*) by computer. For these vectors and matrices, in addition to the previous expressions of $\boldsymbol{R}_{\mathrm{f}}, E^{j \theta_{1}}, E^{-k \theta_{2}}$ we can select, the normalizing model for $\boldsymbol{C}, \boldsymbol{C}_{1}, \boldsymbol{C}_{2}$ is described as follows again:

$$
\boldsymbol{C}=\left[\rho_{1}, \rho_{2},-\rho_{3}\right]^{\mathrm{T}}
$$

where $\rho_{1}, \rho_{2}$, and $-\rho_{3}$ in Figure 1 are the components of $\mathrm{O}_{1} \mathrm{O}_{2}$ in the direction of $\mathrm{x}_{1}, \mathrm{y}_{1}$ and $\mathrm{z}_{1}$, respectively.

$$
\boldsymbol{C}_{1}=\left[h_{1 x}, h_{1 y}, h_{1 z}\right]^{\mathrm{T}}
$$

where $h_{1 \mathrm{x}}, h_{1 \mathrm{y}}$ and $h_{1 \mathrm{z}}$ are components of any follower displacement $h_{1}$ in space in the direction $\mathrm{x}_{\mathrm{f}}, \mathrm{y}_{\mathrm{f}}$ and $\mathrm{z}_{\mathrm{f}}$, respectively. In general, the coordinate system can be so set that the follower moves along one of the axes. Consequently, the other components will become zero.

$$
\boldsymbol{C}_{2}=\left[h_{2 x}, h_{2 y}, h_{2 z}\right]^{\mathrm{T}}
$$

where $h_{2 \mathrm{x}}, h_{2 \mathrm{y}}$ and $h_{2 \mathrm{z}}$ are the components of any cam displacement $h_{2}$ in the direction of $\mathrm{x}_{2,} \mathrm{y}_{2}$ and $\mathrm{z}_{2}$, respectively.

Apparently, the initial parameters in the design of cam profile are involved in $\boldsymbol{R}_{\mathrm{f}}, E^{j \theta_{2}}, E^{-k \theta_{1}}, \boldsymbol{C}$, $\boldsymbol{C}_{1}, \boldsymbol{C}_{2}$, where the geometric parameters consist of $\mathrm{r}_{\mathrm{f}}, \mathrm{r}_{0}$ and b as well as $\rho_{1}, \rho_{2}$ and $\rho_{3}$; while the kinematical parameters contain $\theta_{2}, h_{2}, \theta_{1}$ and $h_{1}$. Among them, $\theta_{2}$ and $h_{2}$ are the prescribed kinematical parameters of cam .The kinematical parameters $\theta_{1}$ and $h_{1}$ of follower can be achieved by means of the motion specifications designed .It is assumed that the counterclockwise $\theta_{1}$ and $\theta_{2}$ are positive and the initial displacements are covered in four displacement parameters.

The following are several examples for the typical cam mechanisms to illustrate the way in which the initial parameters are chosen in table 1. Only the fixed coordinate systems are plotted for the simplification of the schematic diagrams. Moreover, the selection of $\boldsymbol{R}_{\mathrm{f}}, E^{j \theta_{2}}, E^{-k \theta_{1}}$ stated previously will not be repeated any more, and only the axes represented by the $j$ and $k$ axes for the spatial mechanisms will be shown.

\section{Conclusions}

The unified mathematical model presented in this paper is adaptable for the design of cam profile and the geometric analysis of all types of cam mechanisms. In the meanwhile it can be used to develop the general purpose CAD software for the design and the geometric analysis of cam mechanisms. The diverse cam mechanisms, as long as the initial parameters are adequately chosen, can be well manipulated via the unified mathematical model. 


\section{Acknowledgement}

This paper is supported by the Natural Science Foundation of China (51275210), the Produce-Learn-Research Foundation of Jiangsu Province (BY2013015-30), the Foundation of Nanjing Institute of Industry Technology (YK12-01-04) and the Foundation of student of Jiangsu Province(CX12-SCXT-03).

Table 1 Typical cam mechanisms name and the initial parameters are chosen

\begin{tabular}{|c|c|c|c|}
\hline Cam mechanisms figure & $\begin{array}{l}\text { Mechanisms name and } \\
\text { initial parameters chosen }\end{array}$ & Cam mechanisms figure & $\begin{array}{l}\text { Mechanisms name and initial } \\
\text { parameters chosen }\end{array}$ \\
\hline 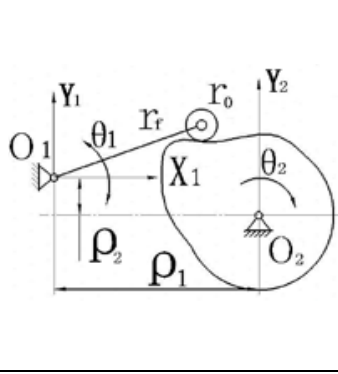 & $\begin{array}{l}\text { Plate cam - oscillating } \\
\text { follower } \\
b=0, \beta=0 \\
\delta_{\mathrm{f}}=0 \\
C=\left[\rho_{1},-\rho_{2}\right]^{T} \\
C_{1}=C_{2}=0\end{array}$ & , & $\begin{array}{l}\text { Globoid cam - oscillating } \\
\text { follower } \\
\begin{array}{l}\boldsymbol{j} \rightarrow \boldsymbol{y}_{2}, \quad \boldsymbol{k} \rightarrow \boldsymbol{x}_{1} \\
r_{f}=0, \beta=90^{\circ} \\
\boldsymbol{C}=\left[0,-\rho_{2},-\rho_{3}\right]^{T}, \boldsymbol{C}_{1}=\boldsymbol{C}_{2}= \\
0\end{array}\end{array}$ \\
\hline 8 & $\begin{array}{l}\text { Plate cam - translating } \\
\text { follower } \\
\begin{aligned} \theta_{1} & =0, \quad r_{\mathrm{f}}=0 \\
b & =0, \quad \beta=0 \\
\delta_{\mathrm{f}} & =0 \\
C & =\left[\rho_{1},-\rho_{2]}\right]^{T} \\
C_{1} & =\left[0, h_{1}\right]^{T} \\
C_{2} & =0\end{aligned}\end{array}$ & & $\begin{array}{l}\text { End cam - oscillating follower } \\
\begin{array}{l}j \rightarrow y_{2}, \quad k \rightarrow x_{1} \\
r_{f}=0, \beta=90^{\circ} \\
C=\left[0,-\rho_{2}, 0\right]^{T} \\
C_{1}=C_{2}=0\end{array}\end{array}$ \\
\hline$Y_{2} Y_{1}$ & $\begin{array}{l}\text { Grooved cam - in-line } \\
\text { follower } \\
\begin{aligned} \theta_{1}=0, \quad r_{\mathrm{f}}=0 \\
b=0, \quad \beta=0\end{aligned} \\
\left.\delta_{\mathrm{f}}=0, \quad C=\left[0,-\rho_{2}\right]\right]^{T} \\
C_{1}=\left[0, h_{1}\right]^{T}, C_{2}=0 \\
\text { Since this cam possesses } \\
\text { the two profiles, so the } \\
\text { value of } \varphi \text { will get "+, -" } \\
\text { from } \phi(\varphi, t)=0\end{array}$ & $Z_{2}$ & $\begin{array}{l}\text { Cylindrical cam - translating } \\
\text { follower } \\
\theta_{2}=0, j \rightarrow y_{2} \\
\beta=90^{\circ} \\
\boldsymbol{C}=\left[0, \rho_{2},-\rho_{3}\right]^{T} \\
\boldsymbol{C}_{1}=\left[0, \boldsymbol{h}_{1}, 0\right]^{T} \\
\boldsymbol{C}_{2}=0\end{array}$ \\
\hline$]\left[\mathrm{E}_{2}\right.$ & $\begin{array}{l}\text { Plate cam - knife-edge } \\
\text { follower } \\
\theta_{1}=0, \quad r_{f}=0 \\
b=0, r_{0}=0, \delta_{f}=0 \\
\boldsymbol{R}_{\mathrm{f}}=0 \\
\boldsymbol{C}=\left[\rho_{1},-\rho_{2}\right]^{T} \\
\boldsymbol{C}_{1}=\left[0,-h_{1}\right]^{T}, \\
\boldsymbol{C}_{2}=0\end{array}$ & $y=\underbrace{}_{X_{2} L}$ & $\begin{array}{l}\text { Conic cam - translating follower } \\
\begin{array}{l}\theta_{1}=0, j \rightarrow z_{2} \\
\beta=\gamma \\
\boldsymbol{C}=\left[0, \rho_{2},-\rho_{3}\right]^{T} \\
\boldsymbol{C}_{1}=\left[0, \boldsymbol{h}_{1} \sin \gamma, \boldsymbol{h}_{1} \cos \gamma\right]^{T} \\
\boldsymbol{C}_{2}=0\end{array}\end{array}$ \\
\hline$\frac{\mathrm{h}_{1}}{\mathrm{O}_{1}\left(\begin{array}{l}\theta_{2} \\
\mathrm{O}_{2}\end{array}\right)}: \mathrm{X}_{2}$ & $\begin{array}{l}\text { Plate cam - flat-faced } \\
\text { follower } \\
\qquad \begin{array}{l}\theta_{1}=0, \quad r_{f}=0 \\
b=0 \\
\delta_{f x}=0 \\
C=\left[\rho_{1},-\rho_{2}\right]^{T} \\
C_{1}=\left[0, h_{1}\right]^{T}, \\
C_{2}=0\end{array}\end{array}$ & $Z_{1} Y_{1}$ & $\begin{array}{l}\text { Cylindrical cam - oscillating } \\
\text { follower } \\
j \rightarrow y_{2}, \quad k \rightarrow z_{1} \\
\beta=90^{\circ} \\
C=\left[0, \rho_{2},-\rho_{3}\right]^{T} \\
C_{1}=C_{2}=0\end{array}$ \\
\hline
\end{tabular}




\section{References}

[1] J.Chakraborty, S.G.Dhande. Kinematics and Geometry of Planar and Spatial Cam Mechanisms; John Wiley Sons, New York, 1977.

[2] Peng Guoxun, Xiao Zhengyang. Design of Cam Mechanisms for Automatic machinery, Beijing, China Machine Press, 1990.

[3] Zhao Han, Olhoff N, Lauristen S. General Analytic Formulas of Kinematical Geometry of Cam Mechanisms, Chinese Journal of Mechanical Engineering, Vol.31, 1995

[4] Changqi Liu, Yeyang Mu and Xijing Cao. Design of Cam Mechanism. Beijing:

China Machine Press, 2005.

[5] Tsay D.M.Wei H.M. A General Approach to the Determination of Planar and Special Cam Profiles, ASME J. of Mech. Des. , Vol. 118,1996 